\title{
Practical tools for implementing early palliative care in advanced lung cancer
}

To the Editor:

We read with interest the article by BLum and SCHÖNFELD [1]. Early introduction of palliative care (EPC) in the management of patients with advanced lung cancer is recommended because it improves quality of life and tends to produce better survival [2]. Hospital-based palliative teams mainly focus on terminal care for hospitalised patients. Involvement of the treating pulmonologist in this setting is warranted, as stated by BLUM and SCHÖNFELD [1]. We would like to show you how we started EPC in collaboration with the palliative team in our hospital (table 1).

We piloted a "palliative hour" to improve symptom control and communication regarding the palliative setting as described in our review [3]. Each patient diagnosed with advanced lung cancer is referred to this outpatient clinic, preferably within 12 weeks from the start of first-line chemotherapy. Making the appointment is already the start of EPC. The meaning of the word "palliative" should be explained by the treating physician, and well understood by the patient and their family. This appointment is often a landmark moment in which the patient and his family realise that the disease is incurable. We have standardised this first consultation, which is led by a pulmonologist who has attended courses in palliative care and communication skills, by a semistructured interview. In Belgium, the treating physicians can reimburse a "longer visit" once for every patient diagnosed with cancer. We use this reimbursement for the first palliative hour, which consists of eight components.

1) Check the readiness of the patient to talk about palliative care by the asking following open questions. What do the patient and their caregivers expect from this consultation? What do they think palliative care is? This gives the opportunity to repeat the diagnosis and explain palliative care and its goals. The emphasis is on the incurability of the disease. A frank prognosis is only given when asked. It is debatable whether a frank prognosis needs to be offered or not. In our opinion, a realistic understanding of the disease and treatment goal (palliation) often leads the patient to ask about his prognosis themselves whenever they are ready for it.

\section{TABLE 1 Local setting}

Type of hospital
City, country
New lung cancer patients per year
Palliative care unit
Palliative team

Thoracic oncology team Palliative hour (EPC)

Practical

Patient EPC course

Patient oncology course

\author{
Academic hospital \\ Antwerp, Belgium \\ $\sim 200$
}

No

Yes, run twice a week by a palliative nurse and a pain physician taking care of all palliative patients in the hospital

They visit on request

3 thoracic oncologists, 1 dedicated nurse, 1 psychologist

Thoracic oncologist: fixed day, once a month

Pain physician: monthly, fixed day

Thoracic oncology nurse: monthly, on a fixed day

Psychologist: daily, on appointment

Palliative nurse: daily, on appointment

Social worker: daily, on appointment

First appointment is always with the pulmonologist followed by a consultation with the palliative care physician 2 months later, with follow-up consultations every second month with the other team members depending on the need of the patient During chemotherapy every week In follow-up every 6-8 weeks

EPC: early introduction of palliative care. 
2) Symptom management is important in palliative care. It is not only about pain and pulmonary symptoms. Psychological symptoms such as sleep and mood disturbances also need to be checked with the patient and treated. At this moment, we plan to use the European Organisation for Research and Treatment of Cancer Quality of Life Questionnaire (QLQ)-LC13 and QLQ-C15-PAL to assess the multidimensional complaints.

3) Routine check of the patient's wishes concerning end of life (EoL) care is conducted. Most patients and family caregivers are willing to talk about this issue. We start this conversation with open hypothetical questions: "Imagine that your condition worsens due to your cancer and you are not capable of taking care of yourself. Have you already thought about this situation? What would you prefer? Would you stay at home with extra help from others or go to a hospice?". Hypothetical questions are more effective in encouraging the patient to discuss this topic. If a patient is considered not ready to enter this topic, it will not be addressed in this session. It can be too soon for some patients and a new attempt to discuss EoL care will be made in the next session.

4) At the start of palliative home care, the general practitioner (GP) is informed by telephone of the patient's participation in the EPC programme and we ask for the GP's contribution. This means that the GP will contact the palliative network to start nursing aid, depending on the patient's needs. The GP is the coordinator of this palliative home team. We feel that this approach is appreciated by most GPs, as in $40 \%$ of the cases, the palliative network had been contacted before the patient's second appointment.

5) The deliberation of the "do not resuscitate" (DNR) code is performed according to the AND (allow natural death) principle [4]. This emphasises that the order is to allow natural consequences of the disease and the ongoing EoL, which makes this topic easier. The DNR code is discussed and noted in the patient's file.

6) Another member of the palliative team (preferably the palliative care physician or nurse) is introduced to the patient. If the patient needs to be hospitalised later on, the palliative team already knows the patient and their family, which avoids mistakes and redundancy at hospitalisation.

7) We finish by emphasising the fact that we realise this diagnosis is difficult for the patient and their family. A lot is going on, and this can cause psychological distress for both the patient and their family. This is an attempt to estimate the need for and the openness to professional psychological help.

8) After 8 weeks, a follow-up appointment is made with the palliative care physician of the palliative team in order to assess symptom control, to discuss topics that have not yet been covered and to check if palliative home care has already started. After evaluation of this project, we noticed that in only $30 \%$ of the patients, the palliative care team (doctor and nurse) was involved, and then rather late in the course of the disease, so we changed our strategy and involved the palliative care physician at the second appointment. To date, this palliative hour has been introduced to 32 patients. Two patients did not keep this appointment: they felt it was not necessary and too soon to talk about palliative care. Three patients did not attend after making an appointment: one experienced a family crisis, one was hospitalised and the other was enrolled in hospice care. Figure 1 shows the percentage of patients that has been seen by each member of our palliative team.

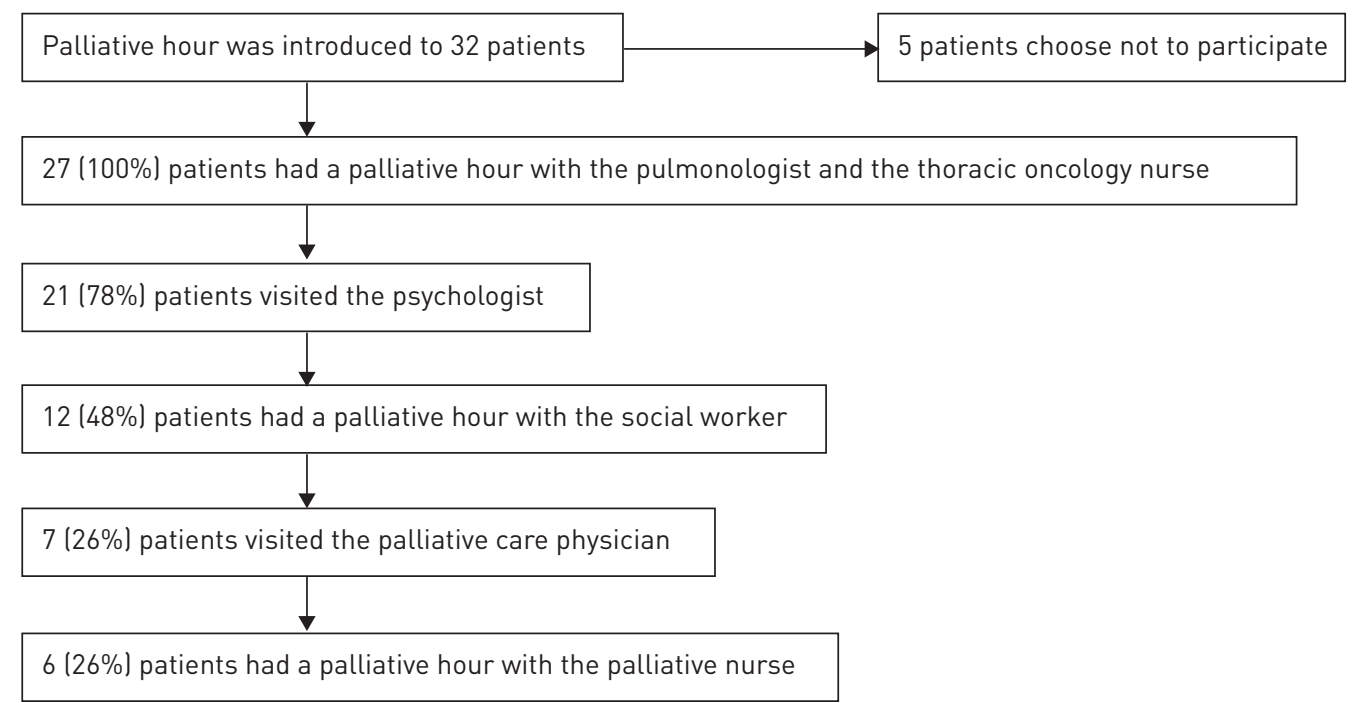

FIGURE 1 Patient disposition in the local pilot study. 
The strength of EPC is its holistic approach. This means that this palliative hour is run not only by the pulmonologist but also by a palliative care physician, a psychologist, a social worker, a palliative nurse and a thoracic oncology nurse. Each team member has their own specific domain to discuss. For example, the palliative nurse will empower the patient to talk about the EoL and give more practical information about the different possibilities, such as home care, hospice care, palliative sedation, etc. Since this palliative hour is implemented in our standard oncology care, short-notice, unplanned appointments are always possible. Specific problems needing another profession are planned in the patients' ambulatory service (e.g. neurologist) and information exchange is linked with the weekly patient discussions.

As mentioned before, the first appointment is always planned with the pulmonologist. This way, we make a clear statement that EPC is integrated into our standard oncology care. The second appointment is with the palliative care physician to discuss topics that have not been covered yet and to check if palliative home care has already started. When making follow-up appointments, we make sure that each team member attends the patient once, to assure better psychosocial support.

In conclusion, we have developed tools for the pulmonologist to implement EPC in daily practice. These tasks mainly consist of assuring adequate symptom control, and transparent communication with patients, relatives and team members. As EPC needs a holistic approach, collaboration with other members of the palliative team is necessary.

@ERSpublications

Pulmonologists need to be involved in early palliative care for patients with metastatic lung cancer http://ow.ly/U3oXg

Annelies Janssens $^{1}$, Liesbeth Teugels ${ }^{1,2}$, Sisca Kohl ${ }^{1,3}$, Toke Michielsen ${ }^{1,3}$, Bert Leysen $^{4}$ and Jan P. van Meerbeeck ${ }^{1}$

${ }^{1}$ Thoracic Oncology, Antwerp University Hospital, Edegem, Belgium. ${ }^{2}$ Multidisciplinary Pain Team, Antwerp University Hospital, Edegem, Belgium. ${ }^{3}$ MOCA, Antwerp University Hospital, Edegem, Belgium. ${ }^{4}$ Primary and Interdisciplinary Care, University of Antwerp, Wilrijk, Belgium.

Correspondence: Annelies Janssens, Thoracic Oncology, Antwerp University Hospital, Wilrijkstraat 10, 2650 Edegem, Belgium. E-mail: annelies.janssens@uza.be

Received: March 092015 | Accepted after revision: Sept 212015

Conflict of interest: None declared.

\section{References}

1 Blum T, Schönfeld N. The lung cancer patient, the pneumologist and palliative care: a developing alliance. Eur Respir J 2015; 45: 211-226.

2 Temel JS, Greer JA, Muzikansky A, et al. Early palliative care for patients with metastatic non-small-cell lung cancer. N Engl J Med 2010; 363: 733-742.

3 Janssens A, Teugels L, Kohl S, et al. Integrating early palliative care (EPC) in the management of lung cancer: the role of the thoracic oncologist. Lung Cancer 2015; 90: 135-138.

4 Levin TT, Coyle N. A communication training perspective on AND versus DNR directives. Palliat Support Care 2014; $28: 1-3$.

\section{Paradoxical decrease in isoprostane and increase in superoxide dismutase following CPAP withdrawal in OSA}

\section{To the Editor:}

STRADLING et al. [1] have recently published an interesting, unprecedented study assessing the effects of a continuous positive airway pressure (CPAP) withdrawal on oxidative stress in patients already treated for moderate-to-severe obstructive sleep apnoea (OSA). Based on a combination of two different studies, the authors showed that stopping CPAP for 2 weeks ("sham-CPAP" group) significantly decreased the urinary F2-isoprostane concentration, while it increased the activity of the blood superoxide dismutase (SOD), 